\title{
Impact of COVID-19 Pandemic on Interventional Radiology Practice-A Multicenter Observational Study
}

\author{
Mathew Cherian ${ }^{1}$ Pankaj Mehta ${ }^{1}$ Sitaram Barath ${ }^{2}$ Manish Yadav ${ }^{3}$ Muthurajan Pandi ${ }^{4}$ \\ Saurabh Joshi ${ }^{5}$ Rahul Kareparambil Ranasingh ${ }^{6}$ Akhil Monga ${ }^{1}$ Karthikeyan Muthugounder Athiyappan ${ }^{1}$ \\ Nikhil Handihal Reddy ${ }^{1}$
}

${ }^{1}$ Department of Interventional Radiology, KMCH Hospital, Coimbatore, India

${ }^{2}$ Department of Interventional Radiology, Geetanjali Medical College and Hospital, Udaipur, India

${ }^{3}$ Department of Interventional Radiology, KIMS Hospital, Trivandrum, India

${ }^{4}$ Department of Interventional Radiology, Ramakrishna Hospital, Coimbatore, India

${ }^{5}$ Department of Interventional Radiology, Vein Center, Mumbai, India

6Department of Interventional Radiology, Government Medical College, Kozhikode, India

J Clin Interv Radiol ISVIR:2020;4:167-174

\author{
Address for correspondence Sitaram Barath, MD, Department of \\ Radiology, Interventional Radiology Subdivision, AS-1 Geetanjali \\ University Campus, Hiran Magri Extension, Near Eklingpura \\ Chouraha, Nh-8 Bypass, Manvakhera, Udaipur, Rajasthan, \\ India 313002 (e-mail: barath.sitaram@gmail.com).
}

\section{Abstract}

Keywords

- COVID-19

- interventional radiology

- impact on health care
Background The COVID-19 pandemic has brought unprecedented challenges to health care services including interventional radiology (IR). Treating COVID-19 infected patients became a priority; furthermore, government policies of differing elective procedures and the public's fear of contacting COVID-19 have impacted IR workload worldwide. The aim of this study was to evaluate the impact of the COVID-19 pandemic on the workflow in six vascular IR centers located across India.

Methods The data were collected retrospectively from April 1 to June 30, 2020. All the six centers were staffed by the alumni of a single parent center located in India. Data was also collected from the same time period in 2019 for comparison.

Results A total of 893 patients were treated from April 1 to June 30, 2019, and 419 were treated during the same period in 2020 during the pandemic, a 53\% case volume reduction (95\% Cl:28. 56-129.44; $p<0.001$ ). The month of April had the largest case volume reduction $(66 \%, 95 \% \mathrm{Cl}$ : $13.57-50.43 ; p<0.001)$. Elective procedures showed an $85 \%$ reduction (95\% Cl: 9.62-91.71; $p<0.001$ ). Venous interventions showed the highest reduction of $76 \%$ (95\% Cl: $0.75-67.75 ; p<0.001)$. Neurological emergencies, dialysis-related interventions, and nonvascular procedures did not show a significant change. No patient tested positive for COVID-19 prior to the procedure; however, one patient who was treated emergently was found to be positive later.

Conclusion COVID-19 pandemic has severely impacted IR practice across India. Workload reduction was more profound at the beginning of the COVID-19 pandemic with a gradual improvement over time.
DOI https://doi.org/

$10.1055 / \mathrm{s}-0040-1718790$

ISSN 2457-0214.
C2020. Indian Society of Vascular and Interventional Radiology.

This is an open access article published by Thieme under the terms of the Creative Commons Attribution-NonDerivative-NonCommercial-License, permitting copying and reproduction so long as the original work is given appropriate credit. Contents may not be used for commercial purposes, or adapted, remixed, transformed or built upon. (https://creativecommons.org/licenses/by-nc-nd/4.0/).

Thieme Medical and Scientific Publishers Pvt. Ltd. A-12, 2nd Floor, Sector 2, Noida-201301 UP, India 


\section{Introduction}

Novel coronavirus or "severe acute respiratory syndrome coronavirus 2 (SARS-CoV-2)" pneumonia first originated in Wuhan, China, in December 2019. ${ }^{1}$ India reported its first case on January 30,2020 , which was followed by a resurgence of cases in the beginning of March. This compelled the federal government of India to impose a nationwide lockdown starting from March 24, 2020, which resulted in a major shift in the pattern of work and hospital working guidelines. Dispensing medical services for both COVID and non-COVID-19 patients became a challenge. Further, protection of the medical workforce treating COVID-19 infected patients became a necessity. ${ }^{2-4}$

Because of a variety of procedures supporting multiple disciplines of medicine, interventional radiology (IR) plays an important role in day-to-day patient care in several difficult situations. ${ }^{5}$ Thus, the workers in this specialty have a substantial risk of contracting and transmitting infection secondary to close contact with patients. ${ }^{6-8}$ IR specific guidelines were put in place to limit the transmission primarily through physical distancing, use of personal protection equipment (PPE), and disinfection. Additionally, specific steps were recommended to optimize resources and manpower. ${ }^{9}$ This resulted in a reduction in workload, affecting some procedures more than the others. We aimed to study the impact of the ongoing COVID-19 pandemic on IR workflow in different IR centers across India during the first 3 months of its spread in India.

\section{Methods}

The study was conducted at six vascular IR centers. These centers were staffed by the alumni of a single center and were located in different geographical areas across India. Data was collected retrospectively for the period from the April 1 to June 30,2020 , during different phases of the government-imposed lockdowns: phase 1 (March 25-April 14), phase 2 (April15-May 3), phase 3 (May 4-17), phase 4 (May 18-31), and unlock 1.0 (June 1-30). Data from April 1, 2019 to June 30, 2019 was also retrieved for comparison. All vascular and nonvascular invasive diagnostic and therapeutic procedures, central line or dialysis catheter insertion, and image-guided procedures such as biliary and abscess drainages were included in the study.

Image-guided biopsies and fine needle aspiration cytology (FNAC) were excluded due to the inability to retrieve comparative data from 2019.

\section{Data Recording}

Retrospective data of the total number of patients treated during the corresponding periods (April 1-June 30) in 2019 and 2020 were retrieved from the medical records and analyzed. Various demographic, clinical, and procedural variables that were included are shown in - Table $\mathbf{1}$. No significant demographic differences were noted during the pandemic as compared with 2019. The mean age was 51 years in 2019 versus 49 years in 2020. No difference was seen in gender proportions: males (54\% reduction) and females (52\% reduction). However, at one center exclusively devoted to the treatment of varicose veins, the patient cohort was younger (mean 33 years during the pandemic vs. 52 years during 2019), as elective procedures were selectively postponed in the elderly patients (age $>60$ years) during the pandemic.

All procedures were categorized according to the Society of Interventional Radiology (SIR) classification of case urgency. ${ }^{10}$

Variation in the number of procedures was analyzed after classifying them based on the month in which it was done, type of procedure, and the involved organ system.

\section{Interventional Radiology (IR) Practice Reorganization}

All patients prior to undergoing any IR procedure were screened for symptoms of COVID-19 infection, such as fever, cough, or symptomatic personal contacts. High-resolution computed tomography (HRCT) chest or reverse transcription polymerase chain reaction (RT-PCR) was done whenever possible before the procedure or according to the local hospital guidelines. HRCT findings were classified according to the COVID-19 Reporting and Data System (CORADS) score. ${ }^{11}$ CORADS 4 and 5 were considered highly suggestive of COVID-19 infection, whereas CORADS 3 was considered suspicious.

All health care workers who were actively involved in treatment compulsorily donned full airborne personal protective equipment (PPE: N95 mask, face shield, gown, and gloves) as per the Indian Council of Medical Research (ICMR) and Ministry of Health and Family Welfare (MOHFW) guideline before entering the angiography room, irrespective of the patient's COVID-19 status. ${ }^{12}$ All patients were made to wear surgical masks to prevent droplet dissemination whenever possible.

In the majority of the cases, local anesthesia or mild sedation was preferred. Patients who required general anesthesia were intubated in emergency or ICU and brought to the interventional suite. If intubation was required during the procedure, it was performed by the attending anesthesiologist with adequate aerosol-preventive measures.

\section{Health Care Worker Surveillance}

The RT-PCR/Tru-NAT test was performed only if a health care worker was symptomatic or had unprotected exposure to a patient with confirmed or suspected COVID-19.

\section{Statistical Analysis}

The data was processed in Microsoft Excel and IBM SPSS Statistics 20.0; statistical averages and relevant proportions were calculated. Confidence intervals were calculated at 95\%. Two-proportion z-test was used for the level of significance ( $p$ values) and the Chi-square test was used for association. The statistical significance threshold was set at $5 \%$. Tables and charts were prepared for simplification and a better understanding of data. 
Table 1 Demographic, clinical and procedure-related parameters

\begin{tabular}{|c|c|}
\hline \multirow[t]{14}{*}{ Parameters } & Age \\
\hline & Sex \\
\hline & Classification of case urgency \\
\hline & Diagnosis \\
\hline & Diagnostic/interventional \\
\hline & Intervention performed \\
\hline & System involved (neuro/GIT/ peripheral vascular/dialysis-related/venous/ aortic/ bleeding) \\
\hline & Vascular/ nonvascular \\
\hline & Technical outcome (success/failure) \\
\hline & Clinical outcome (recovered/ disabled/died) \\
\hline & Pre-procedure test for COVID-19 (PCR/CT chest/others) \\
\hline & Time from admission to procedure (hours) \\
\hline & Covid status \\
\hline & Personal protection (PPE/N 95/N 95 + visor/triple layer mask) \\
\hline \multirow[t]{7}{*}{ Emergent } & All trauma cases \\
\hline & Acute bleeding \\
\hline & Hemodialysis access thrombectomy \\
\hline & Urgent IVC filter placement, DVT endovascular treatment \\
\hline & Sepsis-related interventions (drainage, symptomatic effusions) \\
\hline & Stroke thrombectomy \\
\hline & Subarachnoid hemorrhage, acute limb ischemia \\
\hline \multirow[t]{4}{*}{ Urgent } & $\begin{array}{l}\text { Interventional oncology related treatment (ablation, SIRT, TACE), ports, catheters, biopsy for initial } \\
\text { diagnosis and staging of cancer) }\end{array}$ \\
\hline & Venous access for specific indications (stem cell therapy, intravenous antibiotics, chemotherapy) \\
\hline & Secondary prevention of stroke (carotid/ICAD stenting), AVM/DAVF embolization \\
\hline & Dialysis-related interventions \\
\hline \multirow[t]{8}{*}{ Elective } & Routine drain or line changes \\
\hline & Vein sampling (adrenal or petrosal, etc.) \\
\hline & UFE, gonadal vein embolization \\
\hline & IVC filter retrievals, chronic venous recanalization, EVLT, VenaSeal, sclerotherapy \\
\hline & Arteriovenous or venous malformations \\
\hline & Routine PAD \\
\hline & Research biopsies \\
\hline & Thyroid biopsies, treatment of unruptured aneurysm/AVM \\
\hline
\end{tabular}

Abbreviations: AVM, arteriovenous malformation; DAF, dural arteriovenous fistula; DVT, deep vein thrombosis; EVLT, endovenous laser treatment; GIT, gastrointestinal tract; ICAD, intracranial atherosclerotic disease; IVC, inferior vena cava; SIRT, selective internal radiotherapy; TACE, transarterial chemoembolization; UFE, uterine fibroid embolization.

\section{Results}

A total of 893 patients were treated from April 1 to June 30, 2019, and 419 patients were treated during the corresponding period during the COVID-19 pandemic in 2020, which is a $53 \%$ reduction in workload (95\% CI:28.56-129.44; $p<0.001$ ). The month of April 2020 showed a 66\% reduction in the number of procedures (95\% CI: 13.57-50.43; $p<0.001$ ), the month of May 2020 showed a $54 \%$ reduction $(95 \% \mathrm{CI}$ : $13.18-41.49 ; p<0.001)$, and June 2020 showed a $40 \%$ reduction (95\% CI: 0.01-29.32; $p<0.001$ ) (-Fig. 1, - Table 2).
Emergent procedures showed 45\% reduction (95\% CI: - 5.90$41.90 ; p<0.001$ ), urgent procedures showed $16 \%$ reduction ( $95 \%$ CI: $-16.21-29.54 ; p=0.01)$, and elective procedures showed 85\% reduction, (95\% CI: 9.62-91.71; $p<0.001)$ (- Table 2).

Among the various categories of interventions, venous interventions showed highest (76\%) reduction $(95 \% \mathrm{CI}$ : 0.75-67.75; $p<0.001$ ), bleeding-related interventions showed a $71 \%$ reduction, (95\% CI: 0.75-15.92; $p<0.001)$, and neurointerventions showed a $27 \%$ reduction, (95\% CI: - 3.91-19.91; $p<0.001)$. Dialysis-related interventions showed the lowest $(2 \%)$ reduction, (95\% CI: - 9.07-7.07; $p=1.00)$ (-Fig. 2, - Table 2). 
Chi-square test was done to ascertain association between impact on workload and COVID-19 pandemic. Among the 21 parameters studied, 13 had a $p$ value $<0.05$, showing that the change in workload was indeed due to the pandemic (-Table 3).

Among specific procedures, a few showed marginal increase in numbers, but they were not statistically significant. Acute deep venous thrombosis (DVT) interventions showed a 75\% increase (95\% CI: - 2.35-1.35; $p=0.375)$, stroke thrombectomy showed a $34 \%$ increase (95\% CI: $-3.42-2.12 ; p=0.409$ ), and subarachnoid hemorrhage (SAH)-related interventions

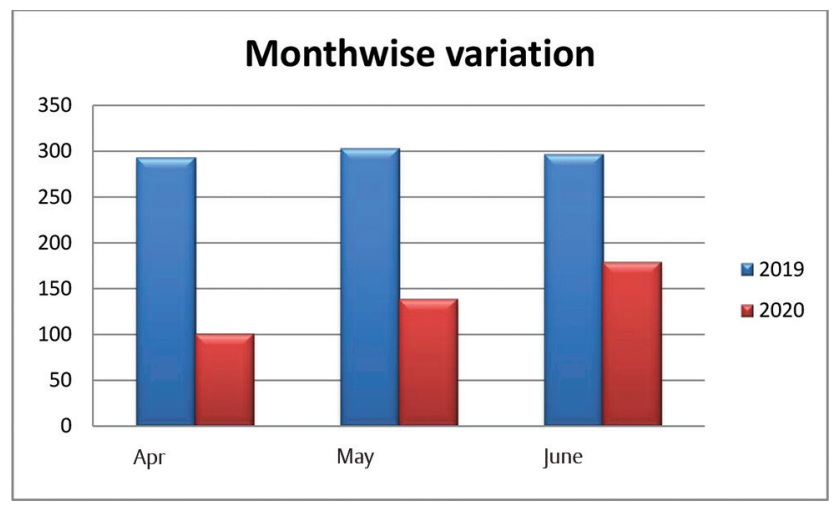

Fig. 1 Shows month-on-month variation in cases before and during the pandemic. Note similar monthly numbers in the year 2019 but a reduction in year 2020 , which gradually improved along with relaxation in lockdown. showed a $17 \%$ increase (95\% CI: - 2.73-1.40; $p=0.625$ ). Procedures such as varicose vein interventions showed the highest (91\%) reduction, (95\% CI: - 7.35-62.35; $p<0.001$ ), followed by transarterial chemoembolization (TACE) for hepatocellular carcinoma, which showed $87 \%$ reduction (95\% CI: 0.09-15.09; $p<0.001$ ) ( - Fig. 3, - Table 4).

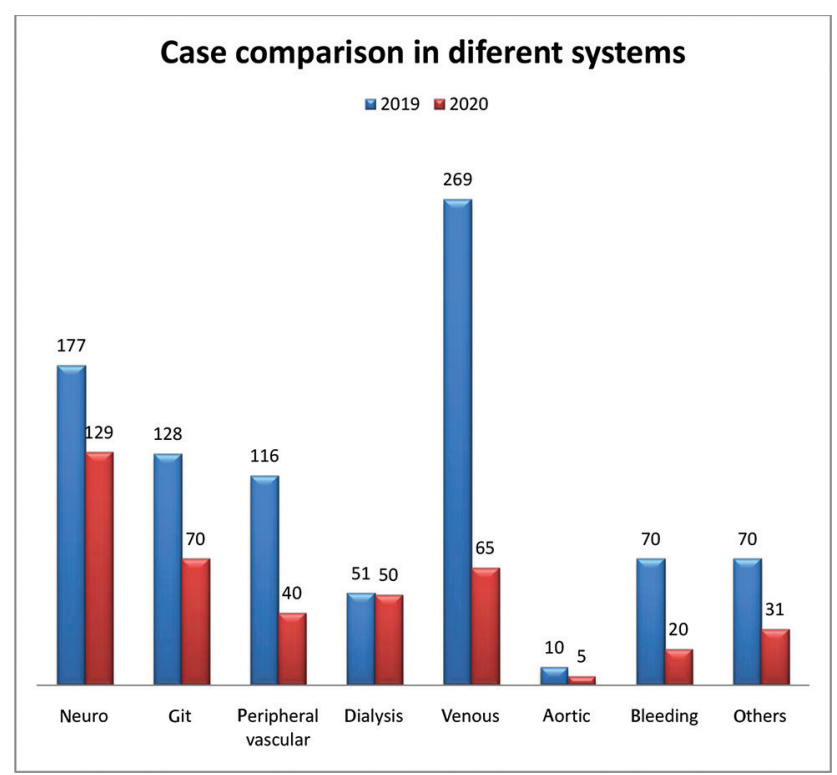

Fig. 2 Shows a comparison in numbers of cases treated from different organ systems during the same period of 2019 and 2020.

Table 2 Comparisonof number of patients among different parameters in COVID-19 and non COVID-19 period

\begin{tabular}{|c|c|c|c|c|c|c|}
\hline & & Total & $\begin{array}{l}\text { Non COVID-19 year } \\
2019\end{array}$ & $\begin{array}{l}\text { COVID-19 year } \\
2020\end{array}$ & Change in \% & $p$-Value \\
\hline \multirow[t]{2}{*}{ Gender } & Male & 828 & 567 & 261 & $-54 \%$ & $<0.001$ \\
\hline & Female & 484 & 326 & 158 & $-52 \%$ & $<0.001$ \\
\hline \multirow[t]{3}{*}{ Month } & April & 394 & 293 & 101 & $-66 \%$ & $<0.001$ \\
\hline & May & 442 & 303 & 139 & $-54 \%$ & $<0.001$ \\
\hline & June & 476 & 297 & 179 & $-40 \%$ & $<0.001$ \\
\hline \multirow[t]{3}{*}{ Urgency } & Emergent & 443 & 286 & 157 & $-45 \%$ & $<0.001$ \\
\hline & Urgent & 454 & 247 & 207 & $-16 \%$ & 0.01 \\
\hline & Elective & 414 & 359 & 55 & $-85 \%$ & $<0.001$ \\
\hline \multirow[t]{2}{*}{ Type } & Diagnostic & 262 & 186 & 76 & $-59 \%$ & $<0.001$ \\
\hline & Interventional & 977 & 644 & 333 & $-48 \%$ & $<0.001$ \\
\hline \multirow[t]{8}{*}{ System } & Neuro & 306 & 177 & 129 & $-27 \%$ & $<0.001$ \\
\hline & GIT & 198 & 128 & 70 & $-45 \%$ & $<0.001$ \\
\hline & $\begin{array}{l}\text { Peripheral } \\
\text { vascular }\end{array}$ & 156 & 116 & 40 & $-66 \%$ & $<0.001$ \\
\hline & Dialysis & 101 & 51 & 50 & $-2 \%$ & 1.000 \\
\hline & Venous & 334 & 269 & 65 & $-76 \%$ & $<0.001$ \\
\hline & Aortic & 15 & 10 & 5 & $-50 \%$ & 0.144 \\
\hline & Bleeding & 90 & 70 & 20 & $-71 \%$ & $<0.001$ \\
\hline & Others & 101 & 70 & 31 & $-56 \%$ & $<0.001$ \\
\hline \multirow[t]{2}{*}{ Category } & Vascular & 1151 & 806 & 345 & $-57 \%$ & $<0.001$ \\
\hline & Non-vascular & 161 & 87 & 74 & $-15 \%$ & 0.181 \\
\hline \multicolumn{2}{|l|}{ TOTAL } & 1312 & 893 & 419 & $-53 \%$ & $<0.001$ \\
\hline
\end{tabular}

Note: Values in bold are statistically significant. 
Table 3 Association of number of patients with different parameters in COVID-19 and non COVID-19 period among different hospitals

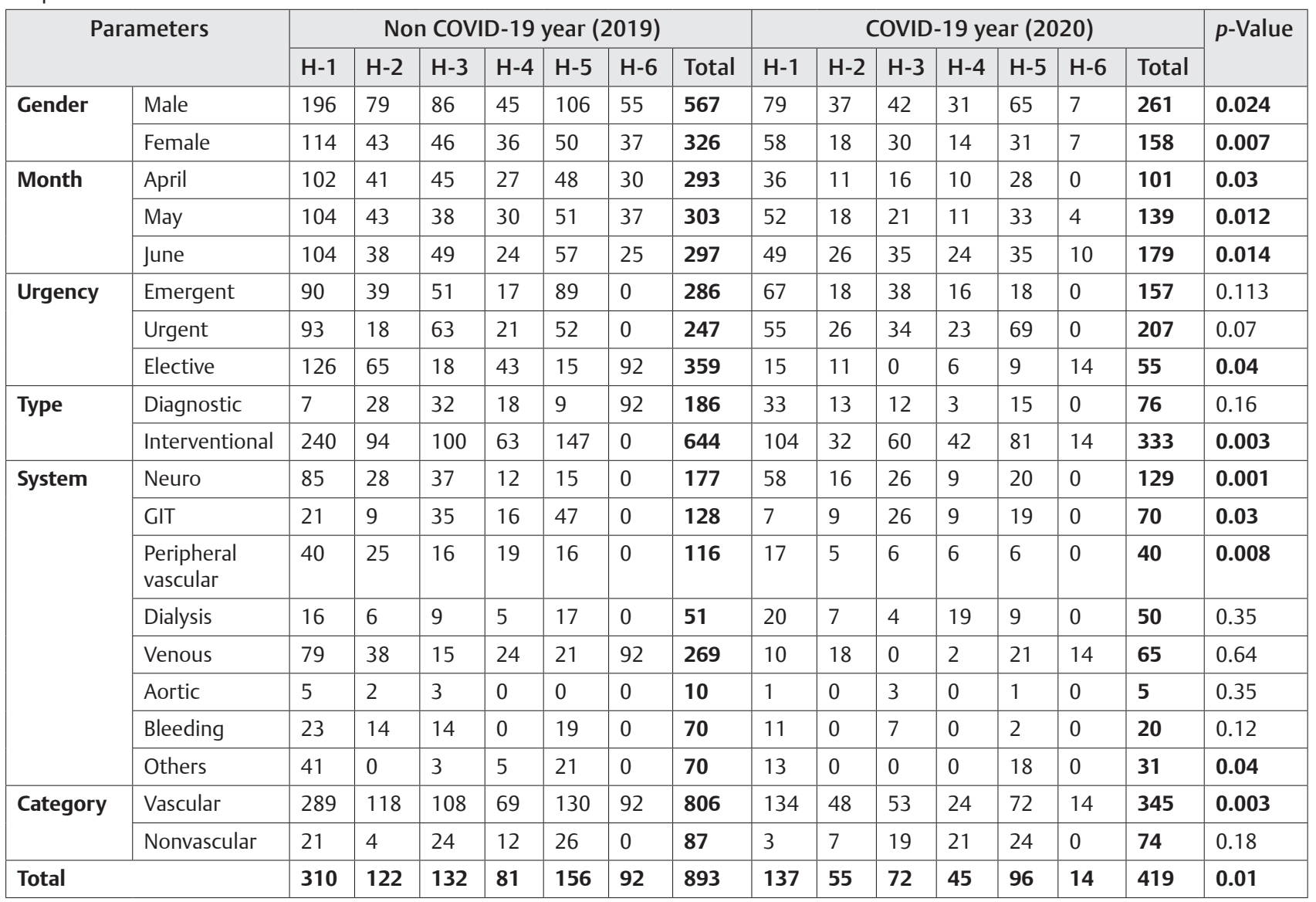

Abbreviation: GIT, gastrointestinal tract.

There was a wide variation in the testing and HRCT protocol among different participating hospitals due to evolving protocols. One hundred and eight patients underwent HRCT thorax and 158 underwent RT-PCR before the procedure. None of the patients had a positive RT-PCR or findings suggestive of COVID-19 infection on CT thorax (CORADS score $\geq 3$ ). Nine health care workers were tested using RT-PCR for interstate travel or due to the presence of mild symptoms and all were negative ( - Fig. 4 ).

\section{Discussion}

The COVID-19 pandemic has brought in unprecedented challenges for medical care in terms of a decrease in workload, infection control, and workforce management. Many recent publications have focused on sharing their experiences and potential challenges relevant to IR services. ${ }^{6,8,13}$ However, to the best of our knowledge, there are no published multicenter data regarding the real impact of the COVID-19 pandemic on IR practice across India.

We included a total of six centers located in different geographical areas within India to understand the overall trend. Five of these centers were providing interventions of all organ systems and were tertiary care hospitals in public (1), private (3), and trust setup (1). One of them was a dedicated varicose vein intervention center. It was important to understand the impact on standalone centers such as varicose vein intervention centers because the procedure per se is often elective.

Our study showed that there was a predictable reduction in the number of IR procedures due to the lockdown. During these 3 months, there was an overall 53\% reduction in work, which was more in April 2020 and slowly improved in May 2020 and June 2020 (66 vs. 54 vs. 40\%). As elective procedures were postponed, the impact was even higher in the elective procedures' subgroup (85\%). A study published by Lezzi et al reported a $48 \%$ overall workload reduction due to the COVID-19 pandemic. ${ }^{14}$

Neurointerventions were among the least affected, as most of these procedures fall under emergencies such as stroke, $\mathrm{SAH}$, and intracerebral hemorrhage. Neurointerventional procedures such as stroke thrombectomy and endovascular therapy for ruptured aneurysm increased marginally (31\%, $p=0.409$ and $14 \%, p=0.625$, respectively). This increase was probably due to the avoidance of an aerosol-generating neurosurgical procedures during the pandemic or closure 


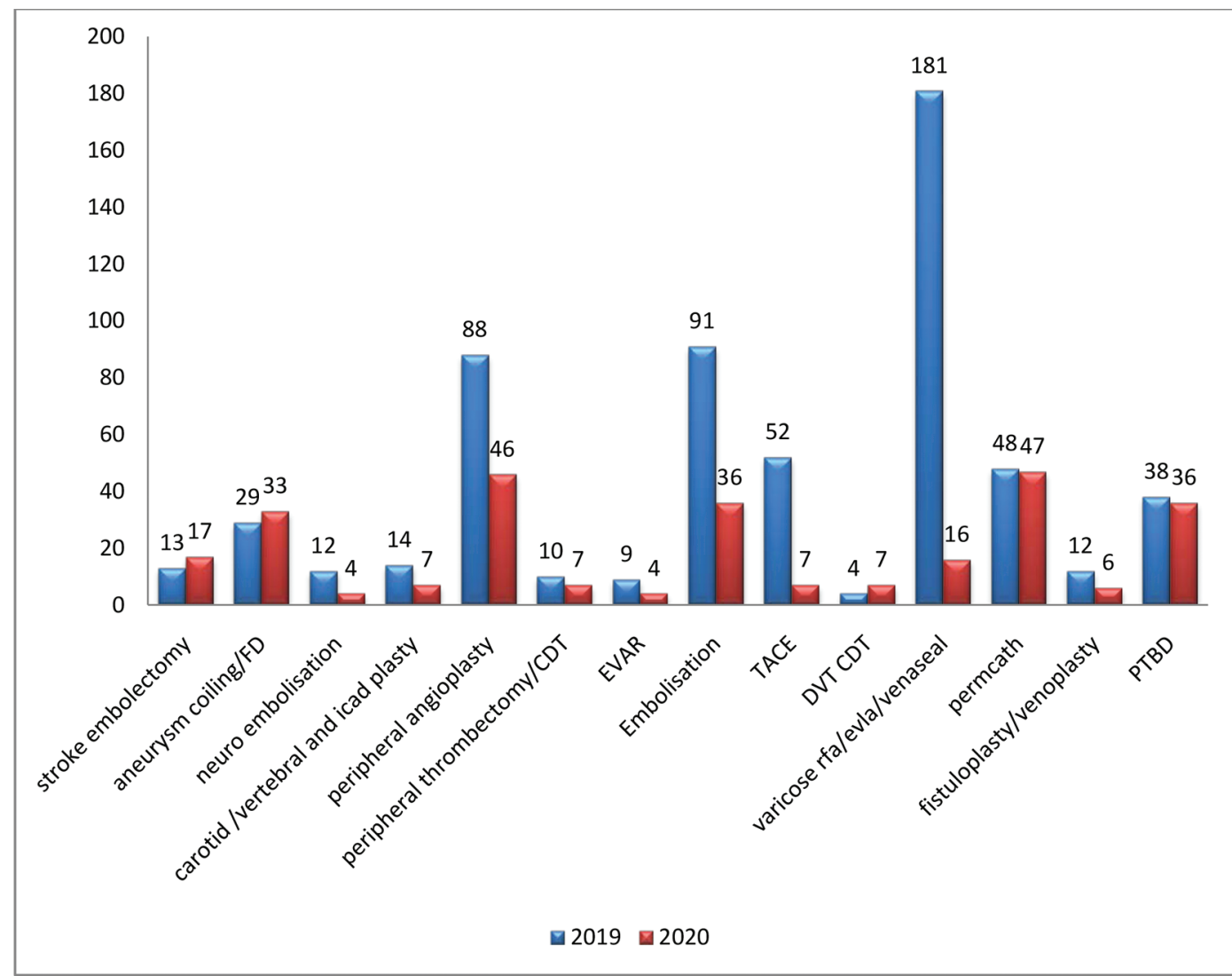

Fig. 3 Shows a comparison in the numbers of different procedures performed during the same period of 2019 and 2020. Not much change was noted in emergency procedures but a significant reduction in elective procedures. CDT, catheter directed thrombolysis; DVT, deep vein thrombosis; EVAR, endovascular aneurysm repair; FD, Flow diverter; PTBD, percutaneous transhepatic biliary drainage; TACE, transarterial chemoembolization.

Table 4 Comparison of number of patients among different vascular intervention in COVID-19 and non COVID-19 period

\begin{tabular}{|c|c|c|c|c|c|}
\hline Procedure & Total & $\begin{array}{l}\text { Non } \\
\text { COVID-19 year } \\
2019\end{array}$ & $\begin{array}{l}\text { COVID-19 year } \\
2020\end{array}$ & Change in \% & $p$-Value \\
\hline Stroke thrombectomy & 30 & 13 & 17 & $31 \%$ & 0.409 \\
\hline Aneurysm coiling/FD & 62 & 29 & 33 & $14 \%$ & 0.625 \\
\hline Neuroembolization & 16 & 12 & 4 & $-67 \%$ & 0.013 \\
\hline Carotid/vertebral and ICAD plasty & 21 & 14 & 7 & $-50 \%$ & 0.05 \\
\hline Peripheral angioplasty & 134 & 88 & 46 & $-48 \%$ & $<0.001$ \\
\hline Peripheral thrombectomy/CDT & 17 & 10 & 7 & $-30 \%$ & 0.512 \\
\hline EVAR & 13 & 9 & 4 & $-56 \%$ & 0.122 \\
\hline Embolization & 127 & 91 & 36 & $-60 \%$ & $<0.001$ \\
\hline TACE & 59 & 52 & 7 & $-87 \%$ & $<0.001$ \\
\hline DVT-CDT & 11 & 4 & 7 & $75 \%$ & 0.375 \\
\hline Varicose RFA/EVLA/VenaSeal & 197 & 181 & 16 & $-91 \%$ & $<0.001$ \\
\hline Permcath & 95 & 48 & 47 & $-2 \%$ & 0.896 \\
\hline Fistuloplasty/venoplasty & 18 & 12 & 6 & $-50 \%$ & 0.088 \\
\hline PTBD & 74 & 38 & 36 & $-5 \%$ & 0.937 \\
\hline Total & 874 & 601 & 273 & $-55 \%$ & $<0.001$ \\
\hline
\end{tabular}

Abbreviations: CDT, catheter-directed thrombolysis; DVT, deep vein thrombosis; EVAR, endovascular aneurysm repair; EVLA, endovenous laser ablation; FD, flow diversion; ICAD, intracranial atherosclerotic disease; PTBD, percutaneous transhepatic biliary drainage; RFA, radiofrequency ablation; TACE, transarterial chemoembolization.

Note: Values in bold are statistically significant. 


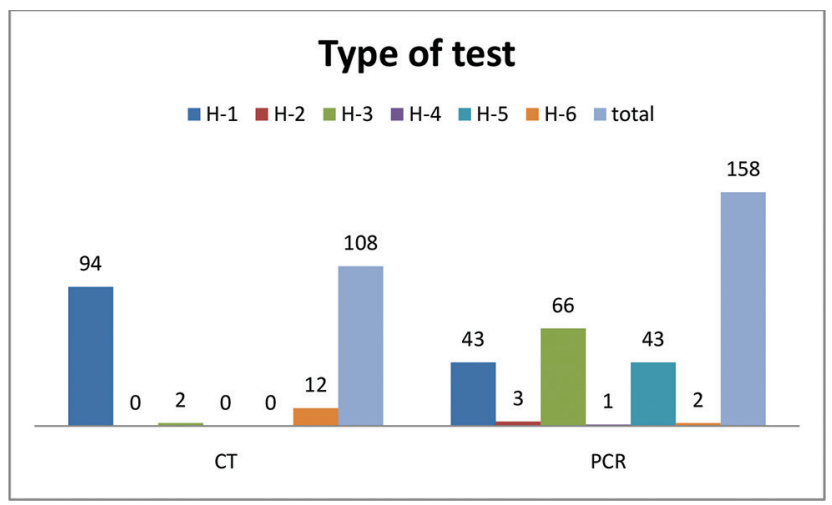

Fig. 4 Shows the number of patients who underwent pre-procedure CT or RT-PCR to diagnose COVID-19 positivity. Note the variation in testing strategy among different hospitals.

of smaller centers. The number of elective neurointerventional procedures such as carotid or vertebral stenting $(33 \%, p=0.05)$ and embolization $(50 \%, p=0.013)$ decreased significantly.

There was overall significant reduction in peripheral vascular interventions $(48 \%, p<0.001)$ and peripheral embolization $(43 \%, p<0.001)$ but no significant change in the number of acute limb ischemia $(17 \%, p=0.512)$. As expected, elective venous interventions showed the highest reduction $(76 \%, p<0.0010)$, especially varicose vein interventions $(91 \%$, $p<0.0010)$. Acute DVT treatment increased by $75 \%$ but was not statistically significant $(p=0.375)$, probably due to selective referral or year-on-year variability. We did not see any case related to COVID-19 hypercoagulability.

Dialysis-related procedures did not show a significant change $(1 \%, p=1.00)$, as these were essential services. Nonvascular procedures also did not show a significant reduction $(8 \%, p=0.181)$, as these were essential services.

A significant association $(p<0.05)$ was found between the COVID-19 pandemic and the total workload, elective procedures, interventional procedures, and vascular procedures. The change in workload was probably not due to other variables, for example, year-on-year variation, change in referral pattern, or change in the reputation of a particular hospital.

\section{Limitations}

The main limitation of our study is the nonuniformity of preprocedure COVID-19 testing. This was due to the limited availability of test kits and laboratories and stricter regulations by the government. Another problem is getting a test result early, especially in emergency cases, as in most of the centers, the results took more than 24 hours. In the beginning, emergency cases were done purely on clinical judgment without HRCT or RT-PCR.

The second limitation of the study is that no COVID-19 positive patient was treated during the study period. This could be because the geographical areas included in this study were in the initial phase of the disease spread and the relatively few positive patients were treated in specially designated hospitals. Hence, the risk of infection to health care workers could not be estimated.

The third limitation is a potential selection bias, as these centers are all staffed by the alumni of one center; hence, the repertoire of the cases may represent a similar practice pattern and need not necessarily represent the overall national trend.

The final limitation of the study is that we compared the data only with that from the previous year. A longer term comparative data would be more useful to differentiate the normal practice variation and the effect of the COVID-19 pandemic. However, our study, by showing the differences with a high statistical significance demonstrated that the changes encountered are less likely to be from practice variation.

\section{Suggestions and Recommendations}

As this pandemic is probably not ending any time soon and there are limited guidelines for health care operations during this crisis, IR services have to continuously care for patients..$^{15}$ IR facilities across India, where most hospitals have a single interventional radiologist with few supporting staff, have additional challenges in terms of delivering the essential services to patients while safeguarding the health care workers. Safety can be improved by making required changes in IR workflow, reducing contamination, performing bedside USG-guided procedures whenever possible, limiting movement or transportation of suspected or positive patients out of their room, ${ }^{16}$ judicious testing, and strict adherence to using appropriate PPE. ${ }^{17-19}$ By following these guidelines, cross-infection among patients and health care workers in the IR department can be minimized. ${ }^{20}$

\section{Conclusion}

During the early months of the COVID-19 pandemic, we observed a significant reduction in the number of procedures performed by IR practices across the country. Although our initial results showed a gradual recovery of case volumes, a longer term study spanning the entire duration of the pandemic would be helpful to understand the broader impact of the pandemic on the IR practice.

\section{References}

1 Wu Y, Ho W, Huang Y, et al. SARS-CoV-2 is an appropriate name for the new coronavirus. Lancet 2020;395(10228):949-950

2 Zhou F, Yu T, Du R, et al. Clinical course and risk factors for mortality of adult inpatients with COVID-19 in Wuhan, China: a retrospective cohort study. Lancet 2020;395(10229):1054-1062

3 Carenzo L, Costantini E, Greco M, et al. Hospital surge capacity in a tertiary emergency referral centre during the COVID-19 outbreak in Italy. Anaesthesia 2020;75(7):928-934

4 Boccia S, Ricciardi W, Ioannidis JPA. what other countries can learn from Italy during the COVID-19 pandemic. JAMA Intern Med 2020;180(7):927-928

5 Charalel RA, McGinty G, Brant-Zawadzki M, et al. IR delivers high-value health care and is an Imaging 3.0 vanguard. J Am Coll Radiol 2015;12:501-506 
6 Chandy PE, Nasir MU, Srinivasan S, Klass D, Nicolaou S, Babu SB. IR and COVID-19: evidence based measures to limit transmission. Diagn Interv Radiol 2020;26:236-240

7 Zhu HD, Zeng CH, Lu J, Teng GJ. COVID-19: what should interventional radiologists know and what can they do? J Vasc Interv Radiol 2020;31(6):876-881

8 Ierardi AM, Wood BJ, Gaudino C, et al. How to handle a COVID-19 patient in the angiographic suite. Cardiovasc Intervent Radiol 2020;43(6):820-826

9 Hoe Gan W, Wah Lim J, Koh D. Preventing intra-hospital infection and transmission of COVID-19 in healthcare workers. Saf Health Work 2020 (e-pub ahead of print). doi: 10.1016/j. shaw.2020.03.001

10 Society of IR - Covid-19 case classification. Available at: https:// www.sirweb.org/practice-resources/toolkits/covid-19-toolkit/ covid-19-case-classification/. Accessed September 23, 2020

11 Prokop M, van Everdingen W, van Rees Vellinga T, et al; COVID-19Standardized Reporting Working Group of the Dutch Radiological Society. CO-RADS: A Categorical CT Assessment Scheme for Patients Suspected of Having COVID-19-Definition and Evaluation. Radiology 2020;296(2):E97-E104

12 Ministry of Health and Family Welfare Directorate General of Health Services [Emergency Medical Relief] Novel Coronavirus Disease 2019 (COVID-19): Guidelines on Rational use of Personal Protective Equipment. Available at: http://www. mohfw.gov.in/pdf/Guidelines on rational use of Personal Protective Equipment.pdf. Accessed September 23, 2020

13 Gogna A, Punamiya S, Gopinathan A, et al. Preparing IR for COVID-19: The Singapore Experience. J Vasc Interv Radiol 2020;31(6):869-875
14 Iezzi R, Valente I, Cina A, et al. Longitudinal study of IR activity in a large metropolitan Italian tertiary care hospital: how the COVID-19 pandemic emergency has changed our activity. Eur Radiol 2020 (e-pub ahead of print). doi: 10.1007/ s00330-020-07041-y

15 Roda WC, Varughese MB, Han D, Li MY. Why is it difficult to accurately predict the COVID-19 epidemic? Infect Dis Model 2020;5:271-281

16 Da Zhuang K, Tan BS, Tan BH, Too CW, Tay KH. Oldthreat, new enemy: is your IR service readyfor the coronavirus disease 2019? Cardiovasc Intervent Radiol 2020;43:665-666

17 Pedicelli A, Valente I, Pilato F, Distefano M, Colosimo C. Stroke priorities during COVID-19 outbreak: acting both fast and safe. J Stroke Cerebrovasc Dis 2020;29(8):104922

18 Ma QX, Shan H, Zhang HL, Li GM, Yang RM, Chen JM. Potential utilities of mask-wearing and instant hand hygiene for fighting SARS-CoV-2. J Med Virol 2020 (e-pub ahead of print). doi: $10.1002 / \mathrm{jmv} .25805$

19 Nicola M, O'Neill N, Sohrabi C, Khan M, Agha M, Agha R. Evidence based management guideline for the COVID-19 pandemic - Review article. Int J Surg 2020;77:206-216

20 World Health Organization. Rational use of personal protective equipment for coronavirus disease (COVID - 19) World Health Organization - Interim Guidance. Available at: https://apps. who.int/iris/bitstream/handle/10665/331215/WHO-2019nCov-IPCPPE_Use-2020.1-eng.pdf. Accessed April 10, 2020 